\title{
Comparison of the effects of long-term pimobendan and benazepril administration in normal cats
}

\author{
Yuichi MIYAGAWA ${ }^{1) *}$, Noboru MACHIDA ${ }^{2)}$, Noriko TODA ${ }^{1)}$, Yoshinori TOMINAGA ${ }^{1)}$ and Naoyuki TAKEMURA ${ }^{1)}$ \\ ${ }^{1)}$ Division of Therapeutic Sciences I, Department of Veterinary Clinical Medicine, School of Veterinary Medicine, Faculty of Veterinary \\ Science, Nippon Veterinary and Life Science University, 1-7-1 Kyonan Cho, Musashino City, Tokyo 180-8602, Japan \\ ${ }^{2)}$ Department of Veterinary Clinical Oncology, Tokyo University of Agriculture and Technology, 3-5-8 Saiwai-cho, Fuchu, Tokyo \\ 183-8509, Japan
}

(Received 18 December 2014/Accepted 2 March 2016/Published online in J-STAGE 14 March 2016)

ABSTRACT. Pimobendan (PIMO) can cause adverse effects, such as mitral valve degeneration, in dogs; however, it is unclear whether these effects occur in cats. Therefore, we aimed to determine whether PIMO or benazepril produces adverse cardiac effects in healthy cats. This was a blinded, randomized, prospective parallel study. Twelve cats were randomly divided into two groups of six cats, namely, an angiotensin-converting-enzyme inhibitor group that received benazepril and a PIMO group. Cats were administered their respective treatments for 506 days, and we evaluated cardiac parameters, blood biochemistry and glomerular filtration rates during that time. At the end of the trial, the cats were euthanized, and histopathological examinations were performed by a pathologist who was blinded to the treatment groups. No significant changes were observed in any of the parameters measured in either of the groups. In particular, no significant cardiac lesions were observed in either of the groups. In healthy cats, neither PIMO nor benazepril appears to cause cardiac lesions, but future studies are needed to examine the effects of PIMO in cats with heart disease.

KEY WORDS: angiotensin-converting-enzyme inhibitor, healthy cat, mitral valve, pimobendan

doi: 10.1292/jvms.14-0673; J. Vet. Med. Sci. 78(7): 1099-1106, 2016

Pimobendan (PIMO) is a benzimidazole-pyridazinone derivative that produces positive inotropic effects by altering intracellular calcium levels and sensitizing myocardial contractions, which causes vasodilatation by inhibiting phosphodiesterase III activity [22]. An important advantage of PIMO is that it increases cardiac output without increasing myocardial oxygen demand. PIMO is used for the management of congestive heart failure $[5,8,11,12,15,18,19]$ and for dilated cardiomyopathy and mitral insufficiency in dogs, and it has been shown to improve clinical signs and survival time $[8,18]$. There is also some evidence that PIMO may have efficacy for managing heart failure in cats $[7,9,13,16]$, considering that it has shown potential to improve cardiac contractility in the presence of cardiac systolic dysfunction. Although hypertrophic cardiomyopathy is the most common heart disease in cats and is characterized by diastolic dysfunction, some cats do develop end-stage dilated hypertrophic cardiomyopathy, characterized by systolic myocardial failure. In this latter setting, PIMO appears to be effective. Indeed, in one retrospective study on cats with various heart diseases, the use of PIMO was well-tolerated [13], and in another study of cats with dilated cardiomyopathy, it was

\footnotetext{
*Correspondence to: Miyagawa, Y., Laboratory of Veterinary Integrative Medicine, School of Veterinary Medicine, Faculty of Veterinary Science, Nippon Veterinary and Life Science University, 1-7-1 Kyonan Cho, Musashino City, Tokyo 180-8602, Japan. e-mail: ymiyagawa@nvlu.ac.jp

(C)2016 The Japanese Society of Veterinary Science

This is an open-access article distributed under the terms of the Creative Commons Attribution Non-Commercial No Derivatives (by-nc-nd) License $<$ http://creativecommons.org/licenses/by-nc-nd/4.0/>.
}

shown to extend the median survival time [9]. In a recent study, the addition of PIMO to the standard treatment was shown to prolong the survival time for cats with congestive heart failure caused by hypertrophic cardiomyopathy [16].

Despite the benefits of treatment, adverse effects have been reported with the use of PIMO in dogs [4, 17, 21]. In a case report of two dogs with mitral insufficiency, a long-term administration of PIMO worsened mitral regurgitation and ventricular hypertrophy [21]. Similarly, in dogs with mild mitral insufficiency, the long-term administration of PIMO was shown to worsen mitral regurgitation and valve lesions [12]. Adverse effects on mitral valves were considered to occur because of increasing cardiac contraction, but it was unclear whether PIMO directly affected the mitral valves. Studies in cats treated with PIMO have not described such adverse cardiac effects [9-11], but this may be because its use in cats is less common. Therefore, the possible adverse cardiac effects of PIMO should be investigated before its use becomes more widespread in cats with cardiac disease.

We aimed to determine whether the long-term administration of PIMO in healthy cats produced adverse cardiac effects, as determined by clinical and histopathological evaluation.

\section{MATERIALS AND METHODS}

Animals: This study was conducted in compliance with the guidelines for the care and use of laboratory animals, and the protocol was approved by the Animal Experimentation Committee of Nippon Veterinary and Life Science University (Approval Number 10-83). Twelve mixed-breed male and female cats (age, $2.2 \pm 0.3$ years; range, $1.7-2.5$ years; 
and weight, $3.3 \pm 0.9 \mathrm{~kg}$ ) were used. Before experimentation, all cats underwent general clinical examination, blood and serum biochemical analyses, electrocardiography, radiography and echocardiography. All cats were considered clinically healthy on the basis of these examinations. Cats were housed in individual cages and fed a commercial maintenance diet (Royal Canin male care and female care, Tokyo, Japan). Water was available ad libitum.

Study design: The study design was based on a similar study conducted in dogs [4]. Twelve cats were randomly assigned into two groups of six. One group received the angiotensin-converting-enzyme inhibitor (ACEI), benazepril (Fortekor, Elanco Animal Health, Kobe, Japan) and was labeled the ACEI group; and the other group received PIMO (Vetmedin, Boehringer Ingelheim Vetmedica, Inc., Tokyo, Japan) and was labeled the PIMO group. Each group was composed of an equal number of animals of both sexes ( 3 males and 3 females). Cats in the ACEI group received benazepril at a dosage of $0.25 \mathrm{mg} / \mathrm{kg} \mathrm{q} 24 \mathrm{hr}$ at 08:00 am for 506 days. Cats in the PIMO group received PIMO at a dosage of $0.25 \mathrm{mg} / \mathrm{kg} \mathrm{q} 12 \mathrm{hr}$ at 8:00 am and 8:00 pm for 506 days.

All cats underwent cardiac auscultation, heart rate measurement, echocardiography, Doppler examination and blood pressure measurement. We also performed hematocrit measurement and serum biochemical analyses that included total protein, albumin, blood urea nitrogen, creatinine, sodium and potassium levels. All analyses were performed before drug administration (day 0) and at days 16, 50, 118, 202, 320, 409 and 506 after starting drug administration. Plasma iohexol clearance (PCio) was performed to measure the glomerular filtration rate (GFR) on days $0,125,323$ and 506. All examinations were performed to assess whether cardiac pathological findings occurred in connection with hemodynamic and cardiac structural changes. Animals were not fed for $8 \mathrm{hr}$ prior to examinations, but were allowed free access to drinking water. To allow the same investigator the time to examine all 12 cats, the examinations were performed between 2:00 pm and 6:00 pm on each assessment day.

Echocardiography and Doppler examination: Cats underwent continuous echocardiography monitoring with an ultrasound unit (Vivid 7, GE Healthcare Japan, Tokyo, Japan) equipped with a 3.5-6.9 $\mathrm{MHz}$ transducer, without sedation or anesthesia. The same trained investigator (Takemura) performed all examinations to exclude possible inter-observer variability, and all measurements were obtained from three consecutive cardiac cycles within the same time frame. The ratio of the left atrial diameter to the aortic diameter was obtained from the right parasternal short-axis view using the B-mode method. The thickness of the anterior and posterior mitral valve leaflet was measured using the left apical four-chamber view, whereas end-diastolic interventricular septum thickness, end-diastolic left-ventricular internal dimension, end-diastolic left-ventricular free-wall thickness and fractional shortening (FS) were measured from the right parasternal short-axis view using the M-mode method (the leading edge to leading edge method). FS was calculated according to the following equation: $F S(\%)=[($ diastolic left-ventricular internal diameter-systolic left-ventricular internal diameter)/diastolic left-ventricular internal diameter] $\times 100$. Any development of mitral valve or tricuspid valve regurgitation was monitored by Doppler examination.

Measurement of PCio: PCio values were measured as previously described [14]. All cats were well-hydrated and had fasted for $12 \mathrm{hr}$ before measurements. Iohexol (Omnipaque 300, Daiichi Sankyo Co., Ltd., Tokyo, Japan) was administered at a dosage of $90 \mathrm{mg}$ iodine $/ \mathrm{kg}$ via the cephalic vein (time 0), and heparinized blood was sampled at 120, 180 and 240 min after administration. Plasma iodine concentrations were determined by the cerium arsenate colorimetric method. The area under the curve (AUC) was estimated from the slope (a) and intercept (A) of the elimination phase of the curve, as determined by linear regression analysis of the final three plasma samples. Clearance $(\mathrm{Cl})$ values were calculated using the following formula: $\mathrm{Cl}(\mathrm{ml} / \mathrm{min})=$ dose of iohexol/AUC, where $\mathrm{AUC}=\mathrm{A} / \mathrm{a}$. The PCio values were then calculated as follows [2]: PCio $(\mathrm{ml} / \mathrm{min})=(0.990778 \times \mathrm{Cl})-$ $\left(0.001218 \times \mathrm{Cl}^{2}\right)$, with the PCio values standardized for body weight.

Measurement of blood pressure: Blood pressure was measured non-invasively by the oscillometric method using a hemomanometer (BP100D, Fukuda M-E Kogyo Co., Ltd., Tokyo, Japan) according to the 2007 American College of Veterinary Internal Medicine guidelines [3]. Cats were individually housed in dimly lit rooms and calmed. Blood pressure was measured repeatedly until stable values were obtained.

Necropsy examination and histopathology: All cats were euthanized by intravenous administration of sodium pentobarbital and potassium chloride on day 507. Histopathological examinations were performed by a veterinary pathologist (Machida) who was blinded to the treatment groups. A complete post-mortem examination was performed on each cat immediately after euthanasia. Tissue samples collected at necropsy were fixed in $10 \%$ neutral buffered formalin and processed using routine methods for paraffin embedding. We then stained $5-\mu \mathrm{m}$ thick sections with hematoxylin and eosin for light microscopy. Selected sections of cardiac tissue, including the septal and parietal valve leaflets of the mitral valve and myocardia, were stained with Masson's trichrome, periodic acid-Schiff, alcian blue (pH 2.5) and toluidine blue.

Statistical analysis: Statistical analyses were performed using Dr. SPSS II (IBM, Tokyo, Japan). Parameters were not normally distributed by Kolmogorov-Smirnov analysis and were expressed as medians (25th and 75th percentiles). Comparison between the baseline parameters of both groups was performed using the Mann-Whitney $U$ test. Changes in parameters measured after drug administration were evaluated by repeated measures analysis of the variance with a split plot design. This test was conducted with the observation day as the intra-subject factor and the drugs (PIMO and benazepril) and animals as the inter-subject factors. If $P<0.05$ was found for the observation day and drug interactions in the analysis of variance, a post-hoc multiple comparison was conducted using the Mann-Whitney $U$ test with Bonferroni correction. A $P$-value $<0.05$ was considered 
Table 1. Summary of characteristics at day 0 in both groups

\begin{tabular}{lccc}
\hline & ACEI group & PIMO group & $P$ value \\
\hline Body weight $(\mathrm{kg})$ & $3.6(2.6,4.2)$ & $3.1(2.6,3.9)$ & 0.937 \\
Age (year) & $1.8(1.8,1.8)$ & $1.8(1.8,2.3)$ & 0.937 \\
Heart rate $(\mathrm{bpm})$ & $154(136,210)$ & $166(162,180)$ & 0.588 \\
SBP $(\mathrm{mmHg})$ & $113(110,134)$ & $139(129,154)$ & 0.180 \\
DBP $(\mathrm{mmHg})$ & $67(57,85)$ & $82(75,84)$ & 0.380 \\
ALB $(\mathrm{g} / \mathrm{d} l)$ & $2.1(2.0,2.1)$ & $2.1(2.1,2.2)$ & 0.310 \\
ALT $(\mathrm{U} / \mathrm{L})$ & $57.0(51.8,69.8)$ & $82.0(72.5,89.3)$ & 0.093 \\
BUN $(\mathrm{mg} / \mathrm{d} l)$ & $20.6(19.4,22.8)$ & $24.8(22.5,25.4)$ & 0.093 \\
Cre $(\mathrm{mg} / \mathrm{d} l)$ & $0.9(0.8,1.0)$ & $0.9(0.9,1.0)$ & 0.818 \\
Na $(\mathrm{mEq} / l)$ & $156.0(156.0,157.5)$ & $157.5(157.0,158.8)$ & 0.132 \\
K $(\mathrm{mEq} / l)$ & $3.8(3.6,4.0)$ & $3.7(3.5,3.8)$ & 0.818 \\
Cl $(\mathrm{mEq} / l)$ & $122.5(121.3,123.8)$ & $123.0(122.3,124.5)$ & 0.589 \\
PCio $(\mathrm{m} l / \mathrm{min} / \mathrm{kg})$ & $2.9(2.7,3.2)$ & $2.8(2.2,3.9)$ & 0.937 \\
LAAo & $1.4(1.3,1.4)$ & $1.3(1.2,1.3)$ & 0.485 \\
IVSd $(\mathrm{mm})$ & $0.4(0.3,0.4)$ & $0.3(0.3,0.4)$ & 0.132 \\
LVIDd $(\mathrm{mm})$ & $1.6(1.5,1.6)$ & $1.6(1.2,1.7)$ & 0.818 \\
LVPWd (mm) & $0.4(0.4,0.4)$ & $0.3(0.3,0.4)$ & 0.310 \\
FS $(\%)$ & $33.9(33.2,38.3)$ & $37.6(35.0,41.0)$ & 0.394 \\
AML $(\mathrm{mm})$ & $0.1(0.1,0.1)$ & $0.1(0.1,0.1)$ & 0.394 \\
PML $(\mathrm{mm})$ & $0.1(0.1,0.1)$ & $0.1(0.1,0.1)$ & 0.699 \\
\hline
\end{tabular}

Values are presented as medians (25th and 75th percentile). Abbreviations: PCio, Plasma iohexol clearance; LAAo, left atrium to aortic root ratio; IVSd, end-diastolic interventricular septum thickness; LVIDd, end-diastolic left-ventricular internal dimension; LVPWd, end-diastolic left-ventricular posterior wall thickness; FS, fractional shortening; AML, anterior mitral valve leaflet; PML, posterior mitral valve leaflet.

Table 2. Cardiovascular measurements by day of treatment with pimobendan or benazepril

\begin{tabular}{|c|c|c|c|c|c|c|c|c|}
\hline & \multicolumn{8}{|c|}{ Day } \\
\hline & 0 & 16 & 50 & 118 & 202 & 320 & 409 & 506 \\
\hline \multicolumn{9}{|c|}{ Body weight (kg) } \\
\hline ACEI group & $3.7(2.7,4.2)$ & $3.7(2.7,4.2)$ & $3.8(2.6,4.2)$ & $3.8(2.6,4.2)$ & $4.2(3.1,4.6)$ & $4.3(3.2,4.6)$ & $4.2(3.3,4.3)$ & $4.4(3.5,4.5)$ \\
\hline PIMO group & $3.2(2.6,4.0)$ & $3.2(2.6,4.0)$ & $3.2(2.5,4.1)$ & $3.2(2.5,4.2)$ & $3.7(2.8,4.7)$ & $3.7(3.0,4.6)$ & $3.8(2.9,4.6)$ & $3.9(2.9,4.6)$ \\
\hline \multicolumn{9}{|c|}{ Heart rate (bpm) } \\
\hline ACEI group & $155(136,211)$ & $150(139,174)$ & $161(146,201)$ & $165(139,179)$ & NP & $171(153,177)$ & $169(151,191)$ & $185(157,196)$ \\
\hline PIMO group & $166(163,181)$ & $172(138,188)$ & $163(157,175)$ & $170(159,177)$ & NP & $172(170,180)$ & $177(160,195)$ & $188(163,200)$ \\
\hline \multicolumn{9}{|l|}{$\mathrm{SBP}(\mathrm{mmHg})$} \\
\hline ACEI group & $113(111,134)$ & $110(106,122)$ & $126(123,137)$ & $131(129,131)$ & NP & $130(125,138)$ & $138(127,143)$ & $139(135,145)$ \\
\hline PIMO group & $139(130,154)$ & $133(124,147)$ & $130(126,142)$ & $141(128,147)$ & NP & $122(115,147)$ & $136(128,143)$ & $141(127,147)$ \\
\hline \multicolumn{9}{|l|}{ DBP $(\mathrm{mmHg})$} \\
\hline ACEI group & $67(58,85)$ & $59(55,72)$ & $66(63,77)$ & $68(65,76)$ & NP & $76(62,80)$ & $72(67,79)$ & $90(86,93)$ \\
\hline PIMO group & $82(75,84)$ & $68(63,79)$ & $68(66,83)$ & $79(76,84)$ & NP & $87(77,90)$ & $80(77,83)$ & $80(69,95)$ \\
\hline
\end{tabular}

Values are presented as medians (25th and 75th percentile) for body weight, heart rate, and systolic and diastolic blood pressure (SBP and DBP, respectively) in both groups before (day 0) and at days 16, 50, 118, 202, 320, 409 and 506 after drug administration. No significant differences were observed for all parameters between two groups $(P \geq 0.059)$.

statistically significant.

\section{RESULTS}

As shown in Table 1, there were no significant differences in baseline parameters between the groups $(P>0.05)$. Body weight, heart rate, and systolic and diastolic blood pressures did not show significant changes during the 506-day study period, and there were no significant differences between the groups $(P \geq 0.162$; Table 2$)$. In addition, no significant differences were observed for any parameters between the groups during the study $(P \geq 0.093$; Table 3$)$. Specifically, we did not detect any cardiac murmurs, and the hematocrit and serum biochemical parameters remained unchanged and comparable between groups. However, although PCio remained stable for both groups $(P \geq 0.200)$, it was significantly 


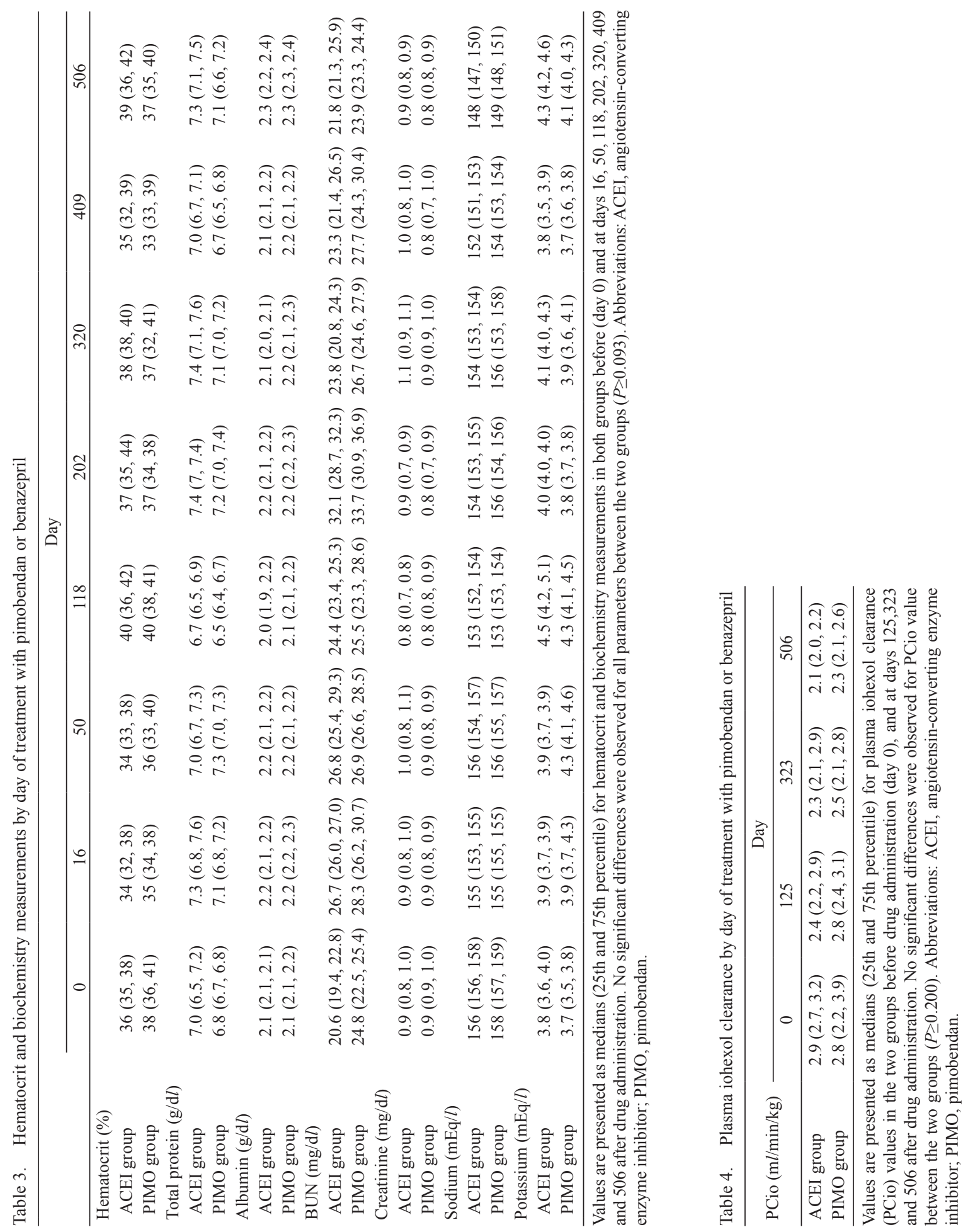




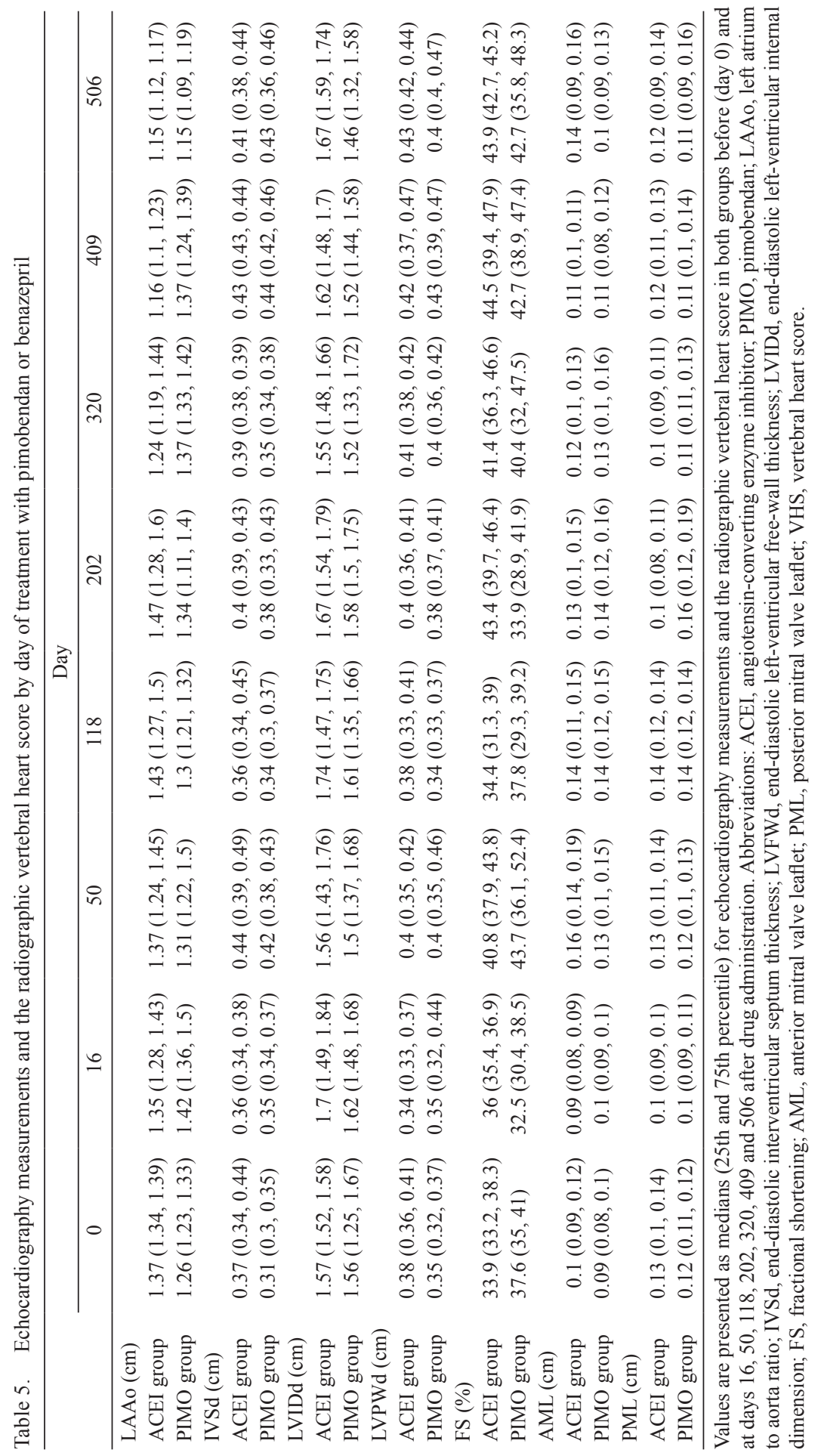



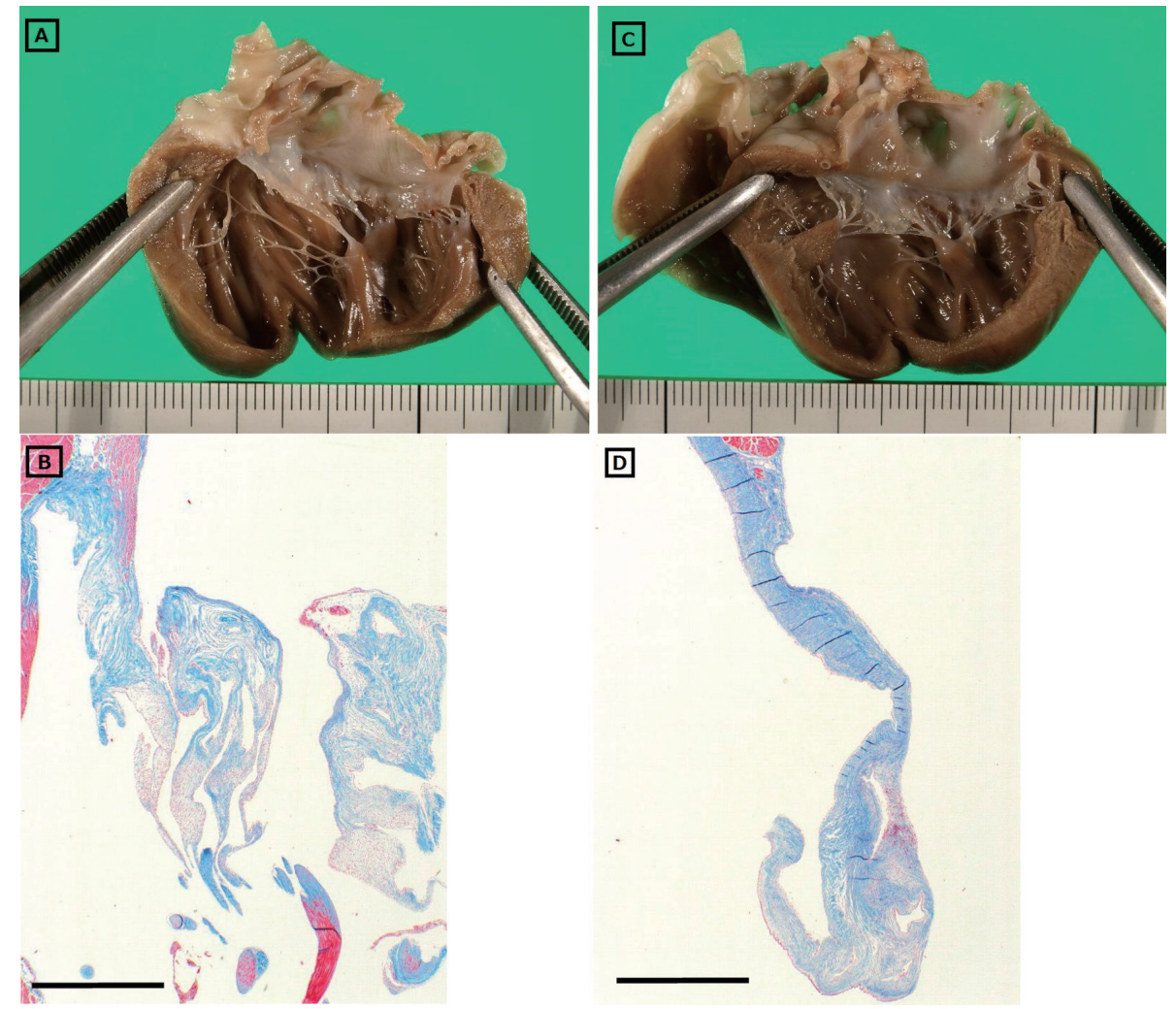

Fig. 1. Macroscopic and histological findings of the mitral valves from cats administered either pimobendan (A \& B) or benazepril (C \& D).

lower at day 506 than at baseline in the ACEI group $(P<0.05$; Table 4). There were no significant changes in echocardiographic measurements during the treatment period (Table 5), and no significant differences were observed between the two treatment groups $(P \geq 0.148)$.

Cats from both groups had evidence of gross cardiac lesions. The septal and parietal valve leaflets of the mitral valve were slightly thickened in three cats in the ACEI group and two cats in the PIMO group (Fig 1). The thickened valves were opaque and white, but the surface was smooth and glistening with no evidence of thrombus formation. Significant gross lesions were not observed in other visceral organs.

All cats had mild-to-moderate histopathological changes in both mitral valve leaflets, with the distal halves being more affected than the proximal halves. The main pathological condition was deposition of acid mucopolysaccharides, primarily within the spongiosa layer of the valve leaflet (Fig 1). Valvular fibrosis was also present, although it was not the predominant histological feature, and inflammatory infiltrates were absent. Therefore, the spongiosa layer was expanded, with focal disruption of the fibrosa by mucopolysaccharide-rich spongiosa, although there were no apparent qualitative or quantitative differences between the groups with respect to the development of these valvular lesions. No other valvular, vascular or myocardial lesions were detected in either of the groups, and no histopathological changes were detected in other visceral organs.

\section{DISCUSSION}

This study demonstrates that long-term administration of PIMO did not produce significant pathological cardiac lesions/adverse effects in healthy cats, which suggests that PIMO might not have the same adverse effects in cats as those reported in dogs [4]. However, although no other valvular, vascular or myocardial lesions were detected, all cats did develop mild-to-moderate myxomatous mitral valve degeneration. The lesions of the mitral valve leaflets included deposition of acid mucopolysaccharides, primarily within the spongiosa layer of the valve leaflet, and valvular fibrosis. All cats in both groups had these changes, and the magnitude of change was equivalent between the two groups.

PIMO and benazepril may not affect cardiac function or hemodynamic status in cats. Indeed, neither PIMO nor benazepril showed any tendency toward a change in echocardiography measurements or GFR during the study, making it unlikely that these caused the myxomatous mitral valve degeneration. In an early study of the incidence of cardiovascular disease in cats, of the 202 cats examined at necropsy, $12(5.9 \%)$ had mitral valve lesions (including endocarditis, fibrosis and stenosis) and 17 (8.4\%) had myocardial lesions (including hypertrophy, hemorrhage, infarct and fibrosis) 
[20]. In our cats, the prevalence of mitral valve degeneration was much higher, but no cat had clinical evidence of mitral regurgitation during the study period. These pathological mitral valve changes were only slight compared with the degree of myxomatous mitral valve degeneration previously observed in dogs. Because no studies have been reported on the development of mitral valve changes with age in healthy cats, it is unclear whether the myxomatous mitral valve degeneration seen in our cats occurred spontaneously, as a natural consequence of aging. A further study is needed to clarify this point.

Cardiomyopathy is the most common cardiac disease in cats. In our study, myocardial lesions were not detected in cats treated with either benazepril or PIMO. In a previous case report of two dogs treated with PIMO, the authors reported that myocardial hypertrophy occurred during treatment of both dogs [21]. However, these dogs were treated with PIMO without any prior echocardiographic examination. Excluding this case report, no adverse effect has been demonstrated conclusively on the myocardia following treatment with PIMO in cats, dogs or humans.

The adverse effect of PIMO on mitral valves in dogs may be caused by increased cardiac contraction, although a direct effect on the valves cannot be excluded. This effect has not been reported in humans, suggesting that there may be species specificity in the pharmacological action of PIMO. It has been reported that, even with the same dose of PIMO administered to cats and dogs, the plasma elimination half-life $(1.3 \pm 0.2 \mathrm{hr}$ and $0.5 \mathrm{hr}$, respectively) and Cmax $(34.5 \pm 6.59 n \mathrm{~g} / \mathrm{m} l$ and $3.09 \pm 0.76 \mathrm{ng} / \mathrm{m} l$, respectively) differed considerably [10]. In addition, the elimination halflife and effect on cardiac contractility of PIMO are simply not associated in dogs [22]. Thus, the tissue concentration and hemodynamic effects of PIMO might be different in cats. In cats with hypertrophic cardiomyopathy, it has been shown that the addition of PIMO to the standard treatment for congestive heart failure can prolong survival times [16]. These cats received a median dose of $0.49 \mathrm{mg} / \mathrm{kg} /$ day $(25 \mathrm{th}$ to 75 th percentile, $0.40-0.67 \mathrm{mg} / \mathrm{kg} /$ day) of PIMO. The dosage used in our study, which was $0.25 \mathrm{mg} / \mathrm{kg}$ twice daily, could therefore be considered sufficient for clinical efficacy in improving cardiac function in cats. However, there was no difference in cardiac changes between the cats administered PIMO (0.25 mg/kg twice daily) and benazepril. It is unlikely that PIMO at $0.25 \mathrm{mg} / \mathrm{kg}$ twice daily has a direct cardiac effect in healthy cats. The present study has shown only that PIMO with clinically effective dose for cats may not have cardiac adverse effect on cats. Extra-cardiac adverse effects of PIMO in cats include salivation, vomiting, agitation, anorexia and constipation $[7,9,13]$. However, no such adverse effects were evident in any cat in this study.

In this study, there were no significant changes in heart rate, blood pressure or GFR after administration of PIMO, suggesting that PIMO does not influence the hemodynamics of healthy cats. In dogs with experimental mitral insufficiency, Kanno et al. reported that PIMO significantly increased renal blood flow, but not GFR [11]. Furthermore, research has shown that PIMO did not alter renal function in healthy dogs [6] and that GFR in dogs receiving PIMO was not significantly different from GFR in dogs receiving benazepril [4]. Unfortunately, renal blood flow was not directly measured in our study.

Our study had some limitations. First, because the optimal dose of PIMO is unknown for cats, we used the same dose of PIMO as that used to treat dogs. In all prior studies into the use of PIMO in cats, the dosage used was $0.25 \mathrm{mg} / \mathrm{kg}$ $\mathrm{q} 12 \mathrm{hr}[7,9,13]$. Given that the plasma elimination half-life and Cmax differ considerably between cats and dogs [10], the required dose and frequency of administration of PIMO in cats may differ from those in dogs. Despite all previous studies on PIMO for cats selected the dosage based on that previously used dosages for dogs $[7,9,13,16]$, no clinical adverse effects were reported. In addition, one study showed that using the same dose of PIMO in cats as in dogs prolonged the survival time in cats with hypertrophic cardiomyopathy [16]. These results suggest that the use of PIMO in cats at the same dosage as that used in dogs does not produce a clinical disadvantage. A second limitation is that cardiac contraction was evaluated using only FS. Other methodssuch as the ejection fraction-may be required to observe the true effect of PIMO on cardiac contraction. In addition, PIMO has been shown to accelerate left-ventricular isovolumetric relaxation and to improve distensibility in conscious dogs with tachycardia-induced heart failure [1]. In our study, evaluation of cardiac relaxation, including the E/A ratio and isovolumetric relaxation time, was not performed. Thus, the cardiac effects of PIMO on echocardiography might not have been accurately assessed. A third limitation is that only healthy cats were used, which contrasts with a prior study in dogs [4]. However, no study has evaluated the adverse effects of PIMO on the mitral valves of healthy dogs. Mitral regurgitation in cats is often caused by systolic anterior motion associated with cardiomyopathy. A further study is justified to evaluate the possible effects of PIMO on mitral regurgitation in cats with cardiomyopathy.

In conclusion, neither PIMO nor benazepril administration was associated with cardiac lesions in healthy cats, suggesting that both agents can be used in cats without the risk of cardiotoxicity. However, future studies are needed to examine the adverse cardiac effects of PIMO in cats with pre-existing heart disease. In addition, there is a need to examine age-related alterations in the hearts of healthy cats.

\section{REFERENCES}

1. Asanoi, H., Ishizaka, S., Kameyama, T., Ishise, H. and Sasayama, S. 1994. Disparate inotropic and lusitropic responses to pimobendan in conscious dogs with tachycardia-induced heart failure. J. Cardiovasc. Pharmacol. 23: 268-274. [Medline] [CrossRef]

2. Bröchner-Mortensen, J. 1972. A simple method for the determination of glomerular filtration rate. Scand. J. Clin. Lab. Invest. 30: 271-274. [Medline] [CrossRef]

3. Brown, S., Atkins, C., Bagley, R., Carr, A., Cowgill, L., Davidson, M., Egner, B., Elliott, J., Henik, R., Labato, M., Littman, M., Polzin, D., Ross, L., Snyder, P., Stepien R., American College of Veterinary Internal Medicine 2007. Guidelines for the identi- 
fication, evaluation, and management of systemic hypertension in dogs and cats. J. Vet. Intern. Med. 21: 542-558. [Medline] [CrossRef]

4. Chetboul, V., Lefebvre, H. P., Sampedrano, C. C., Gouni, V., Saponaro, V., Serres, F., Concordet, D., Nicolle, A. P. and Pouchelon, J. L. 2007. Comparative adverse cardiac effects of pimobendan and benazepril monotherapy in dogs with mild degenerative mitral valve disease: a prospective, controlled, blinded, and randomized study. J. Vet. Intern. Med. 21: 742-753. [Medline] [CrossRef]

5. Fuentes, V. L., Corcoran, B., French, A., Schober, K. E., K1eemann, R. and Justus, C. 2002. A double-blind, randomized, placebo-controlled study of pimobendan in dogs with dilated cardiomyopathy. J. Vet. Intern. Med. 16: 255-261. [Medline] [CrossRef]

6. Fusellier, M., Desfontis, J. C., Le Roux, A., Madec, S., Gautier, F., Thuleau, A. and Gogny, M. 2008. Effect of short-term treatment with meloxicam and pimobendan on the renal function in healthy beagle dogs. J. Vet. Pharmacol. Ther. 31: 150-155. [Medline] [CrossRef]

7. Gordon, S. G., Saunders, A. B., Roland, R. M., Winter, R. L., Drourr, L., Achen, S. E., Hariu, C. D., Fries, R. C., Boggess, M. M. and Miller, M. W. 2012. Effect of oral administration of pimobendan in cats with heart failure. J. Am. Vet. Med. Assoc. 241: 89-94. [Medline] [CrossRef]

8. Häggström, J., Boswood, A., O’Grady, M., Jöns, O., Smith, S., Swift, S., Borgarelli, M., Gavaghan, B., Kresken, J. G., Patteson, M., Ablad, B., Bussadori, C. M., Glaus, T., Kovacević, A., Rapp, M., Santilli, R. A., Tidholm, A., Eriksson, A., Belanger, M. C., Deinert, M., Little, C. J., Kvart, C., French, A., Rønn-Landbo, M., Wess, G., Eggertsdottir, A. V., O’Sullivan, M. L., Schneider, M., Lombard, C. W., Dukes-McEwan, J., Willis, R., Louvet, A. and DiFruscia, R. 2008. Effect of pimobendan or benazepril hydrochloride on survival times in dogs with congestive heart failure caused by naturally occurring myxomatous mitral valve disease: the QUEST study. J. Vet. Intern. Med. 22: 1124-1135. [Medline] [CrossRef]

9. Hambrook, L. E. and Bennett, P. F. 2012. Effect of pimobendan on the clinical outcome and survival of cats with non-taurine responsive dilated cardiomyopathy. J. Feline Med. Surg. 14: 233-239. [Medline] [CrossRef]

10. Hanzlicek, A. S., Gehring, R., Kukanich, B., Kukanich, K. S., Borgarelli, M., Smee, N., Olson, E. E. and Margiocco, M. 2012. Pharmacokinetics of oral pimobendan in healthy cats. J. Vet. Cardiol. 14: 489-496. [Medline] [CrossRef]

11. Kanno, N., Kuse, H., Kawasaki, M., Hara, A., Kano, R. and Sasaki, Y. 2007. Effects of pimobendan for mitral valve regurgitation in dogs. J. Vet. Med. Sci. 69: 373-377. [Medline] [CrossRef]

12. Lombard, C. W., Jöns, O. and Bussadori, C. M. 2006. Clinical efficacy of pimobendan versus benazepril for the treatment of acquired atrioventricular valvular disease in dogs. J. Am. Anim. Hosp. Assoc. 42: 249-261. [Medline] [CrossRef]

13. Macgregor, J. M., Rush, J. E., Laste, N. J., Malakoff, R. L., Cunningham, S. M., Aronow, N., Hall, D. J., Williams, J. and Price, L. L. 2011. Use of pimobendan in 170 cats (2006-2010). J. Vet. Cardiol. 13: 251-260. [Medline] [CrossRef]

14. Miyagawa, Y., Takemura, N. and Hirose, H. 2010. Evaluation of a single sampling method for estimation of plasma iohexol clearance in dogs and cats with various kidney functions. $J$. Vet. Med. Sci. 72: 271-278. [Medline] [CrossRef]

15. O'Grady, M. R., Minors, S. L., O'Sullivan, M. L. and Horne, R. 2008. Effect of pimobendan on case fatality rate in Doberman Pinschers with congestive heart failure caused by dilated cardiomyopathy. J. Vet. Intern. Med. 22: 897-904. [Medline] [CrossRef]

16. Reina-Doreste, Y., Stern, J. A., Keene, B. W., Tou, S. P., Atkins, C. E., DeFrancesco, T. C., Ames, M. K., Hodge, T. E. and Meurs, K. M. 2014. Case-control study of the effects of pimobendan on survival time in cats with hypertrophic cardiomyopathy and congestive heart failure. J. Am. Vet. Med. Assoc. 245: 534-539. [Medline] [CrossRef]

17. Reinker, L. N., Lee, J. A., Hovda, L. R. and Rishniw, M. 2012. Clinical signs of cardiovascular effects secondary to suspected pimobendan toxicosis in five dogs. J. Am. Anim. Hosp. Assoc. 48: 250-255. [Medline] [CrossRef]

18. Summerfield, N. J., Boswood, A., O'Grady, M. R., Gordon, S. G., Dukes-McEwan, J., Oyama, M. A., Smith, S., Patteson, M., French, A. T., Culshaw, G. J., Braz-Ruivo, L., Estrada, A., O’Sullivan, M. L., Loureiro, J., Willis, R. and Watson, P. 2012. Efficacy of pimobendan in the prevention of congestive heart failure or sudden death in Doberman Pinschers with preclinical dilated cardiomyopathy (the PROTECT Study). J. Vet. Intern. Med. 26: 1337-1349. [Medline] [CrossRef]

19. Suzuki, S., Fukushima, R., Ishikawa, T., Hamabe, L., Aytemiz, D., Huai-Che, H., Nakao, S., Machida, N. and Tanaka, R. 2011. The effect of pimobendan on left atrial pressure in dogs with mitral valve regurgitation. J. Vet. Intern. Med. 25: 1328-1333. [Medline] [CrossRef]

20. Tashjian, R. J., Das, K. M., Palich, W. E., Hamlin, R. L. and Yarns, D. A. 1965. Studies on cardiovascular disease in the cat. Ann. N. Y. Acad. Sci. 127: 581-605. [Medline] [CrossRef]

21. Tissier, R., Chetboul, V., Moraillon, R., Nicolle, A., Carlos, C., Enriquez, B. and Pouchelon, J. L. 2005. Increased mitral valve regurgitation and myocardial hypertrophy in two dogs with long-term pimobendan therapy. Cardiovasc. Toxicol. 5: 43-51. [Medline] [CrossRef]

22. van Meel, J. C. and Diederen, W. 1989. Hemodynamic profile of the cardiotonic agent pimobendan. J. Cardiovasc. Pharmacol. 14 Suppl 2: S1-S6. [Medline] [CrossRef] 